\title{
Promising converters of wind and solar energy into electric energy for electrotechnical complexes
}

\author{
Yakov Mikhailovich Kashin \\ Department of Electrical Engineering and Electrical \\ Machines \\ Kuban State Technological University \\ Krasnodar, Russia \\ E-mail: jlms@mail.ru
}

\author{
Mikhail Sergeyevich Khristoforov \\ Department of Electrical Engineering and Electrical \\ Machines \\ Kuban State Technological University \\ Krasnodar, Russia \\ E-mail: khristoforov87@mail.ru
}

\author{
Alexander Valeryevich Samorodov \\ Department of Electrical Engineering and Electrical \\ Machines \\ Kuban State Technological University \\ Krasnodar, Russia \\ E-mail: Alex.Samorodoff@gmail.com \\ Vladislav Anatolievich Kim \\ Department of Electrical Engineering and Electrical \\ Machines \\ Kuban State Technological University \\ Krasnodar, Russia \\ E-mail: vladk-kub@mail.ru
}

\begin{abstract}
The study prospects and reasons the application of wind and solar energy for power supply of industrial facilities. It also analysis current converters of wind and solar energy. The authors provide original designs of a wind-solar generator, axial two-input electrical generator machine, synchronized axial twoinput generator for power supply of local and remote facilities (in terms of power mains accessibility), which enable production of electric energy by concurrent conversion of kinetic wind energy and solar photo energy. The developed systems are capable of working both autonomously and in parallel with industrial threephase AC power lines. Moreover, the elaborated systems allow improving the mass and dimensional characteristics of laboratory test beds, reducing their cost, simplifying their design, enhancing general reliability, reducing energy losses, thus increasing the efficiency factor.
\end{abstract}

Keywords-axial electrical machines, stator, rotor, mass and dimensional charactersitics, magnetic conductor, energy efficiency, synchronization.

\section{INTRODUCTION}

Over the recent years, the utilization of renewable energy sources (RES) and alternative green energy have been drawing more and more attention, which is conditioned by several factors. On the one hand, there is the understanding that natural resources are limited, and reserves of fossil fuel will eventually come to an end. On the other hand, application of conventional (non-renewable) energy leads to increasingly evident ecological consequences (environmental pollution, increased carbon dioxide emission, global warming, etc.) [1$3]$.

Among all RESs in the world, the first place in terms of implementation is occupied by wind energy generation; the second place is taken by solar energy generation. In terms of solar power supply, the lead is taken by solar heat energy supply. Today, $471 \mathrm{M} \mathrm{m}^{2}$ of solar water heaters (helioplants) operate globally with installed power of $330 \mathrm{GW}$ and annual heat energy generation of $281 \mathrm{GWh}$. Currently, the installed power of helioplants in Russia is $8.76 \mathrm{MW}\left(12,514 \mathrm{~m}^{2}\right)$. They are build primarily in three regions: Krasnodar Krai, Buryatia and the Far East. The solar radiation conditions in our country allow increasing the area of helioplants up to $10 \mathrm{M} \mathrm{m}^{2}$. Most actively, the solar heat supply systems are built in Krasnodar Krai $\left(7000 \mathrm{~m}^{2}\right)$. The predicted achievable area is estimated to be $1 \mathrm{M} \mathrm{m}^{2}$ [1].

In this connection, great importance is attributed to the research, development and implementation of converters of solar and wind energy into high-quality electric energy and establishment of electrotechnical complexes on their basis that can work both autonomously and in parallel with the commercial three-phase power mains.

\section{RESULT AND DISCUSSION}

Currently, there is a number of builds of electromechanical converters (hereinafter referred to as EEC) that convert wind and solar energy into electric one [4-8].

Fortis Wind Energy company (Netherlands) have made Fortis Alize wind turbine [5] consisting of fairing, blades, synchronous generator with internal fixed stator with armature magnetic conductor and external rotating rotor with inductor magnetic conductor with radial constant magnets, drive shaft, rotary support, shock absorber and current collector. The blades of the wind turbine are rigidly connected to the rotor front disk.

Fortis Alize wind turbine requires no recurrent maintenance, having no gear and nipples for lubricant refill and equipped with highly durable bearings. The cut-in speed of such turbine is $3.1-4 \mathrm{~m} / \mathrm{s}$; the rated power $(10 \mathrm{~kW})$ is 
achieved at wind speed of $11-15 \mathrm{~m} / \mathrm{s}$. At wind speed more than $16 \mathrm{~m} / \mathrm{s}$, the turbine power abruptly drops due to spontaneous furling of the wind-driven powerplant (WDPP) [5].

This plant generates electric energy only from wind kinetic energy and it cannot generate energy from sunlight.

Simultaneous conversion of mechanical rotation energy (e.g. wind energy) and DC electric energy (e.g., solar energy converted by photovoltaic converters into DC energy) is carried out in a two-dimensional axial electrical generator machine with a wound armature and a DC machine commutator and a rotor with squirrel-cage winding that can freely rotate relative to each other. The rotor and armature are axial, while the armature slots contain AC generator winding which output is connected with AC power line via three slip rings and three brushes.

Such generator machine can combine and convert kinetic wind energy and solar energy generated by photovoltaics into DC electric energy and AC three- (or more) phase electric energy with more stable output parameters as compared to conventional electromechanical energy converters.

Axial design of such generator machine provides maximum possible rotor inertia with minimal waste of smart materials, which is important for smoothing mechanical energy input steps, e.g. in the case of sharp-edged gust, i.e. the axial rotor plays a role of inertial mechanical energy accumulator.

The experiments conducted by the authors have shown high potential of such generators in autonomous power supply system based on complex application of non-conventional renewable energy sources, for instance, solar and wind energy within the same power grid.

A drawback of Fortiz Alize wind turbine [5] and twodimensional axial electrical generator machine is low reliability due to the presence of the current collector with movable contacts and commutator.

This drawback is absent in developed by authors axial twoinput contact-less electrical generator machine (ATCEGM) [9], which is also used for wind and solar energy conversion into electrical energy; the general view of the machine is presented in Fig. 1.



Fig. 1. General view of axial two-input contact-less electrical generator machine

ATCEGM consists of case 1, constant multi-polar magnet 1 of subexciter inductor, side axial magnetic conductor 3 with multi-phase winding 4 of subexciter armature, single-phase excitation winding 5 and additional excitation winding 6, which is coupled with DC source through contacts 19 (Fig. 2), internal axial magnetic conductor 7 with multi-phase winding 8 of exciter armature and single-phase excitation winding 9 of the main generator, side axial magnetic conductor 10 with multi-phase (in Fig. 2, nine-phase) winding 11 of the main generator armature, axle 12 mounted in bearing assemblies 13 and 14 and rigidly connected with constant multi-polar magnet 2 of subexciter inductor through disk 15 and with internal axial magnetic conductor 7 through disk 16 .

The rotor is formed by the elements of subexciter magnetic systems, exciter and main generator of the ATCEGM [9] (constant axial multi-polar magnet 3 of subexciter inductor and axial rotating magnetic conductor 7 with multi-phase winding 8 of exciter armature and single-phase winding 9 of main generator excitation) rigidly fixed of the axle using the disks. The rotor axle is mounted in bearing assemblies 13 .

The rotor rotates relatively to the elements of the magnetic systems of the subexciter, exciter and main generator (side axial magnetic conductor with multi-phase main generator armature winding, internal axial magnetic conductor with multi-phase subexciter armature winding, main and auxiliary single-phase exciter excitation windings) mounted on the case. 
Due to the rotation of the rotor with mounted on it elements of magnetic systems of subexciter, exciter and main generator (constant axial multi-polar magnet of subexciter inductor and axial rotating magnetic conductor with multiphase exciter armature winding and single-phase main generator excitation winding), relative to the elements of magnetic systems of subexciter, exciter and main generator (side axial magnetic conductor with multi-phase main generator armature winding, internal axial magnetic conductor with multi-phase subexciter armature winding, main and auxiliary single-phase exciter excitation windings) mounted on the case, mechanical energy is converted into electric energy (in the subexciter), which in the form of DC voltage is fed to the single-phase exciter excitation winding where magnetic flux is generated.

DC electrical energy (e.g. from photovoltaic converters) flowing in the auxiliary exciter excitation winding generates the magnetic flux directed correspondingly to the magnetic flux generated by the single-phase exciter excitation winding. In line with the superposition principle of magnetic fields, the magnetic fluxes generated by the main and auxiliary exciter excitation windings combine. The total magnetic flux interacts with multi-phase exciter multi-phase winding and induces in it a multi-phase EMF system which is rectified by multi-phase full-wave rectifier and fed to single-phase excitation winding of the main generator. Magnetic flux of the single-phase excitation winding of the main generator interacts with multiphase main generator armature winding and induces there a multi-phase EMF system which is supplied to the power line.

Thus, this machine also combines the mechanical energy of rotation and DC energy at the input, converts them and outputs total AC electrical energy.

An appreciable advantage of such axial contact-less electrical generator machine is a significant reduction in its cost as compared to conventional radial electrical machines due to simplified magnetic conductor production technology, less complex winding technology and reduced consumption of electrical steel for magnetic conductors [10].

Another electrical machine that also has no movable contacts and is widely used in electrotechnical complexes, converting wind and solar energy into electric energy, is developed by the authors axial two-input contact-less windsolar generator (ATCWSG) (general view is depicted in Fig. 2).

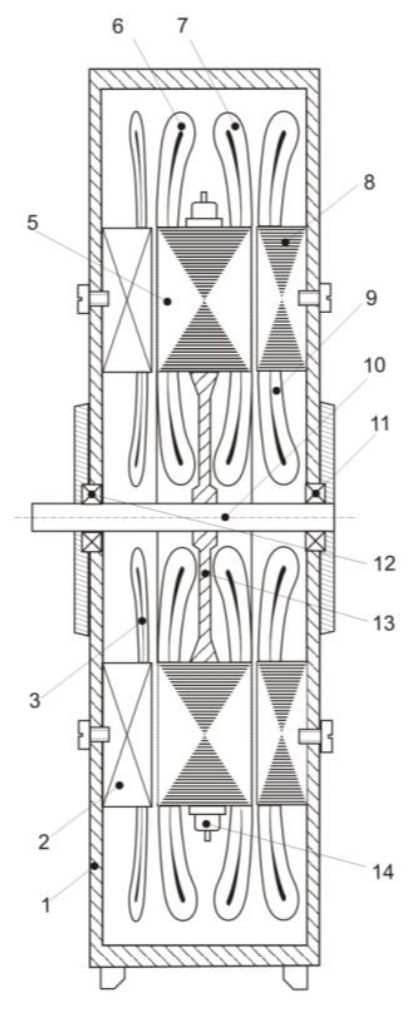

Fig. 2. General view of axial two-input contact-less wind-solar generator

The generator contains exciter and main generator mounted on a single shaft 10 . The exciter consists of exciter inductor and axial magnetic conductor 5 with winding 6 of exciter armature. The main generator consists of side axial magnetic conductor 8 with one active end face, with slots with laid in multi-phase winding 9 of main generator armature, and internal axial magnetic conductor 5 with two active end faces with slots from the side of side axial magnetic conductor with laid in excitation winding 7 of main generator. Side axial magnetic conductor 8 with one active end face is rigidly set in case 1 , while the internal axial magnetic conductor 5 with two active end faces is mounted on shaft 10 capable of rotating relative to side axial magnetic conductor with one active end face. The exciter inductor is made from constant multi-polar magnet 2 and auxiliary exciter excitation winding 3; the constant multi-polar magnet 2 of exciter inductor has slots, several sections and is immovable and rigidly fixed in case 1 , while auxiliary winding 3 of exciter excitation is laid in slots between the sections of constant multi-polar magnet 2 of exciter inductor and coupled with DC source. Internal axial magnetic conductor 5 with two active end faces with slots is mounted in case 1 between constant multi-polar magnet 2 of exciter inductor with auxiliary winding 3 of exciter excitation and side axial magnetic conductor 8 with one active end face allowing for the rotation relative to constant multi-polar magnet 2 of exciter inductor with auxiliary winding 3 of exciter excitation. Winding 7 of main generator excitation is coupled with multi-phase winding 6 of armature excitation through multi-phase full-wave rectifier 14 . 
In such wind-solar generator, the energy losses as compared to the previous machine are reduced at the expense of reduced number of intermediate electromechanical converters. In particular, the energy in the ATCEGM is converted in three electrical machines: subexciter, exciter and main generator, while in the wind-solar generator, there are only two stages of energy conversion: exciter and main generator. In this connection, along with the decreased nonrational energy losses, the mass and dimensional characteristics of such wind-solar generator also improve with simultaneous simplification of the machine design.

A significant drawback of afore described designs (and a number of other EMECs converting wind and solar energy into electric energy as well) is the dependence of voltage frequency generated by them on the rpm of the elements of magnetic systems of subexciter (if present), exciter and main generator mounted on the rotor relative to the elements of magnetic systems of subexciter (if present), exciter and main generator mounted in a stationary case; the frequency is determined as

$$
f=p \cdot n / 60,
$$

where $p$ is the number of pairs of poles; $n$ is the rotor rpm relative to the stationary case.

The rotor rpm, in its turn, is a function of momentum generated on the shaft of known wind generators by the source of mechanical rotation energy, wind in particular; herewith, the moment on the generator shaft in this case depends on the wind force with is the function of the wind speed.

Since the intensity of mechanical energy transfer to the shaft of the electrical generator machine can be uneven (e.g. due to fluctuating wind speed), the rotor rpm can be variable; hence, the voltage frequency collected from the armature winding of such wind generators (wind turbines) is unstable. This limits the application field of such wind turbines that due to noted peculiarities can be used only for supplying local facilities with low requirements to supply voltage and without parallel connection to external three-phase AC systems.

Thus, the afore described and a number of other wind turbines cannot work in parallel with external three-phase AC system.

Moreover, some of them are incapable of directly converting light energy (e.g. solar energy) into DC energy for consequent combination of the energy with mechanical rotation energy and conversion of total energy into electric one, which also limits their application.

Currently, the problems of synchronization of electrotechnical complexes converting solar and wind energy into electric energy with the industrial three-phase AC grid are understudied.

To extend the application field of afore discussed EMECs, the authors have developed synchronized axial two-input generator (SATG) which can operate as a component of an electrotechnical complex in parallel with external three-phase AC system [11].
Fig. 3 depicts a general view of the developed synchronized axial two-input generator; Fig. 4 illustrates its electric diagram.

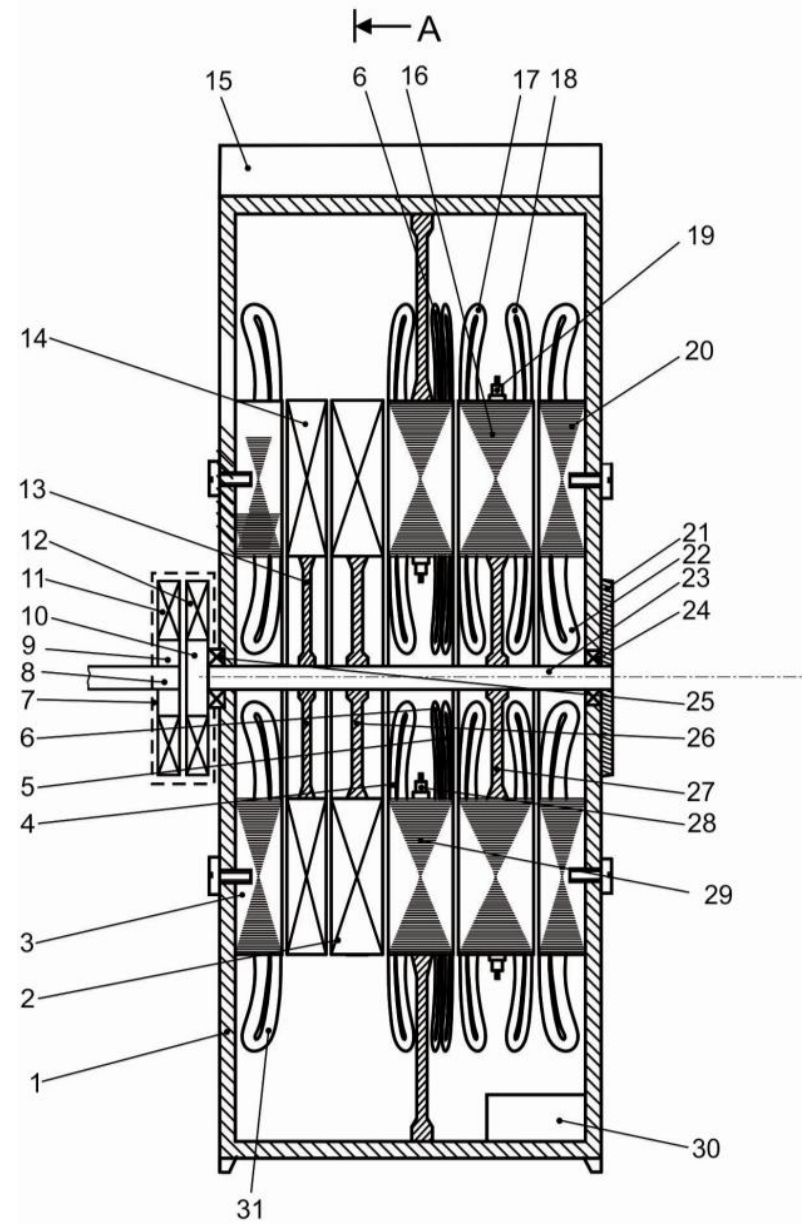

Fig. 3. General view of synchronized axial two-input generator

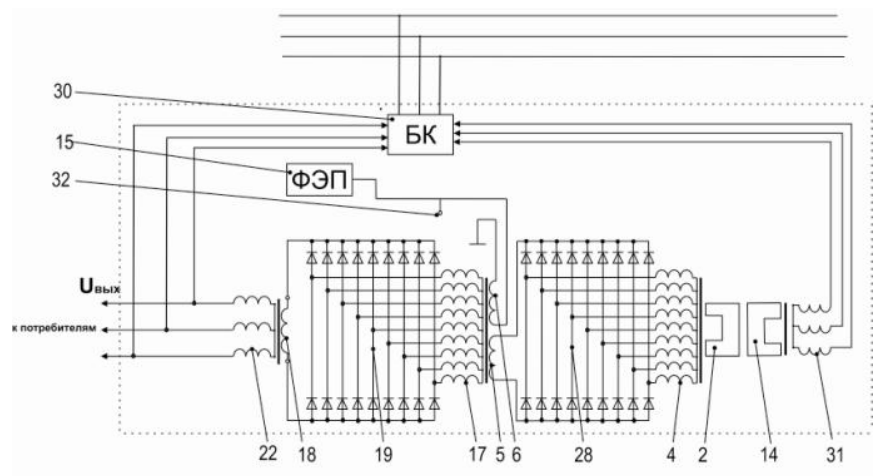

Fig. 4. Electric diagram of synchronized axial two-input generator

The synchronized axial two-input generator (SATG) (Figs. 3 and 4) consists of case 1 with side axial magnetic conductor 20 with three-phase winding 22 of main generator armature, internal axial magnetic conductor 29 with multi-phase winding 4 of subexciter armature, main 5 and auxiliary 6 single-phase exciter excitation windings and rotor with shaft 23 on which disks 26 and 27 are used to rigidly mount constant axial multi- 
polar magnet 2 of subexciter inductor and axial rotating magnetic conductor 16 with multi-phase winding 17 of exciter armature and single-phase winding 18 of main generator excitation. Rotor shaft 23 is mounted on bearing assemblies 24 and 25 and closed by lid 21 from one side and outstands from case 1 from the other side. Single-phase winding 18 of main generator excitation is coupled with multi-phase winding 17 of exciter armature through multi-phase full-wave rectifier 19. Main winding 5 of exciter excitation is coupled with multiphase winding 4 of subexciter armature through multi-phase full-wave rectifier 28 .

In the top part of case 1, photovoltaic cell 15 (PVC) is mounted coupled with auxiliary single-phase winding 6 of exciter excitation which enables coupling to an external photovoltaic cell (not shown in Figs. 3 and 4) through contacts 32 (Fig. 4).

On the end of rotor shaft 23 outstanding from case 1, magnetic gear 7 is mounted which consists of shaft 8 of magnetic gear, driver disk 9 and driven disk 10 made from non-magnetic material and constant magnets 11 and 12 .

In case 1 of the SATG, a voltage synchronizer is mounted which consists of axial magnetic conductor 3 with one active end face with slot with laid in three-phase synchronization winding 31 and constant axial multi-polar magnet 14 rigidly fixed by disk 13 on shaft 23 between axial magnetic conductor 3 of the synchronizer and constant axial multi-polar magnet 2 of subexciter inductor; axial magnetic conductor 3 of the synchronizer is rigidly fixed in case 1 . The distribution of phases of three-phase synchronization winding 31 is made to coincide with the distribution of phases of three-phase winding 22 of main generator armature.

In the bottom part of case 1 , switching unit 30 with two inputs is mounted. To the first input, three-phase winding 22 of main generator armature is coupled; to the second input, three-phase synchronization winding 31 is coupled, while the output enables the connection to the external three-phase AC system. The ends of phases of three-phase synchronization winding 31 enable the coupling with the same ends of the phases of three-phase winding 22 of main generator armature through switching unit 30.

The SATG operates as follows. The SATG rotor is rotated by external source of mechanical energy (input of mechanical energy) - wind, for instance-through magnetic gear 7.

The rotation of shaft 8 of magnetic gear 7 , due to force interaction of constant magnets 11 and 12 induced by the attraction of opposite poles, generates torque which through driven disk 11 is transferred to SATG rotor shaft 23. This torque induces the rotation of constant axial multi-polar magnet 2 of subexciter inductor, which is rigidly fixed on shaft 23 by disk 26 and axial rotating magnetic conductor 16 rigidly fixed on shaft 23 by disk 27, with multi-phase winding 17 of exciter armature and single-phase winding 18 of main generator excitation.

On the rotation of constant axial multi-polar magnet 2 of subexciter inductor and axial rotating magnetic conductor 17 with multi-phase winding 17 of exciter armature and singlephase winding 18 of main generator excitation, the magnetic flux of constant axial multi-polar magnet 2 of subexciter inductor interacts with multi-phase winding 4 of subexciter armature laid in the slots of internal axial magnetic conductor 29 rigidly fixed in case 1 and induces in it a multi-phase EMF system which is rectified by multi-phase full-wave rectifier 28 and fed to the main single-phase winding 5 of exciter excitation laid in the slots of internal axial magnetic conductor 29. Herewith, in the main single-phase winding 5 of exciter excitation, a magnetic flux is generated.

Simultaneously, in PVC 15 (and external PVC, if present) (power line input), light energy is converted into DC electric energy. The direct current flowing in auxiliary single-phase winding 6 of exciter excitation (Fig. 2) generates magnetic flux co-directed with the magnetic flux generated by main single-phase winding 5 of exciter excitation.

The total magnetic flux generated by main 5 and auxiliary 6 single-phase windings of exciter excitation interacts with multi-phase winding 17 of exciter armature laid in the slots of internal axial rotating magnetic conductor 16 and induces multi-phase EMF system in it, which is rectified by multiphase full-wave rectifier 19 and fed to single-phase winding 18 of main generator excitation laid in the slots of axial rotating magnetic conductor 16 .

The magnetic flux of single-phase winding 18 of main generator excitation interacts with three-phase winding 22 of main generator armature laid in the slots of axial magnetic conductor 20 and induces in it a three-phase EMF system which is supplied to consumers and to the first input of switching unit 30 the second input of which is coupled with three-phase synchronization winding 31 laid in the slots of axial magnetic conductor 3 of the synchronizer.

Switching unit 30 couples the SATG (phases of threephase winding 22 of main generator armature) to the external three-phase AC system (to same-name phases of external three-phase AC system), if the following conditions are met:

1. The frequency of the three-phase EMF collected from three-phase winding 22 of main generator armature equals the frequency of three-phase voltage of external three-phase AC system.

2. The magnitude of the three-phase EMF collected from three-phase winding 22 of main generator armature equals the magnitude of three-phase voltage of external three-phase AC system.

3. The phase sequence of three-phase winding 22 of main generator armature and that of three-phase synchronization winding 31 coincides with the phase sequence of external three-phase AC system.

4. Phase coincidence of same-name voltages of the generator and grid.

The output voltage $U_{\text {out }}$ at the output of SATG is synchronized as follows.

With small disturbance momentum, the synchronization momentum generated by three-phase winding 22 of main generator armature is sufficient for the generator to remain in synchronization. 
At large disturbances, a significant desynchronization occurs between voltage phases of voltage generated by threephase winding 22 of main generator armature and voltage of the external three-phase AC system due to different frequencies of output generator voltage collected from threephase winding 22 of main generator armature and the voltage of the external three-phase AC system. In this case, switching unit 30, in which the frequencies of output generator voltage and voltages of external three-phase AC system are compared, couples the phases of three-phase synchronization winding 31 with the external three-phase AC system. Herewith, in threephase synchronization winding 31 , under the action of threephase voltage of external AC system, three-phase electric current flows which generates magnetic field rotating with synchronous frequency.

On the decrease of the frequency of output voltage collected from three-phase winding 22 of main generator armature induced by decreased shaft $23 \mathrm{rpm}$ as compared to voltage frequency of external three-phase AC system, the rpm of synchronizer axial multi-polar magnet 14 rigidly fixed on shaft 23 by disk 13 also decreases. This leads to the shift (advance) of the pole axis of rotating magnetic field of threephase synchronization winding 31 in relation to the pole axes of constant axial multi-polar synchronizer magnet 14. This switches the synchronizer into motor mode, i.e. it consumes active electric energy from external three-phase AC system. In these conditions, the magnetic field generated by electric current flowing in three-phase synchronization winding 31 generates additional torque directed along with the rotor rotation, which increases rotor rpm and the voltage frequency collected from three-phase winding 22 of main generator armature increases up to the voltage frequency of the external three-phase AC system.

On the increase of the frequency of output voltage collected from three-phase winding 22 of main generator armature induced by increased shaft $23 \mathrm{rpm}$ as compared to voltage frequency of external three-phase AC system, the rpm of synchronizer axial multi-polar magnet 14 rigidly fixed on shaft 23 by disk 13 also increases. This leads to the shift (lagging) of the pole axis of rotating magnetic field of threephase synchronization winding 31 in relation to the pole axes of constant axial multi-polar synchronizer magnet 14. This switches the synchronizer into generator mode, i.e. it generates active electric energy, which is fed to the external three-phase $\mathrm{AC}$ system. In these conditions, the magnetic field generated by electric current flowing in three-phase synchronization winding 31 generates additional torque directed opposite the rotor rotation, which decreases rotor $\mathrm{rpm}$ and the voltage frequency collected from three-phase winding 22 of main generator armature decreases down to the voltage frequency of the external three-phase AC system.

Thus, the developed SATG combines the energy input from two different sources: directly converts the light energy fed to the light input (PVC input 9) into DC electric energy, combines it by electromagnetic transformation with mechanical energy received to mechanical input (shaft 23) of the SATG with concurrent conversion of received total energy into electric energy of three-phase AC voltage synchronized in terms of frequency with the voltage of external three-phase AC system.

The synchronized AC voltage is fed to the grid to consumers and to the external three-phase AC system.

Thus, the SATG combines the mechanical and AC electric energy with simultaneous conversion of received total energy into AC electric energy, similarly to ATCEGM [5] and unlike it, due to the capability of direct conversion of light energy and its combination with mechanical energy with consequent conversion of received energy into electric one and capability of connection to external three-phase AC system owing to minimal discrepancy in SATG output voltage frequency and external AC system voltage frequency, and thus allows extending the application sphere of ATCEGMs, i.e. enable direct conversion of light energy and parallel operation of generator and external three-phase AC system.

The capability of direct conversion of light energy into DC electric energy and combination of it with mechanical rotation energy is achieved through the installation of a PVC in the suggested device which is coupled with auxiliary single-phase exciter excitation winding. The light energy is converted by the PVC into DC electric energy. Direct current flowing in the auxiliary single-phase exciter excitation winding generates magnetic flux which as per superposition law combines with the magnetic flux generated with main single-phase exciter excitation winding, which respectively combines the torques generated by currents flowing in the main and auxiliary singlephase exciter excitation windings.

The capability of coupling the SATG with the external three-phase AC system is provided by the installation of the voltage synchronizer, which consists of rigidly fixed in the case axial magnetic conductor with one active end face with slots with laid in three-phase synchronization winding and constant axial multi-polar magnet rigidly fixed by the disk on the shaft between synchronizer axial magnetic conductor and constant axial multi-polar magnet of subexciter inductor. Moreover, the distribution of the phases of three-phase synchronization winding coincides with the distribution of the phases of three-phase winding of the main generator armature and two-input switching unit. To the first input, winding of main generator armature is coupled; to the second input, threephase synchronization winding is coupled, while the output enables the connection to the external three-phase AC system.

The capability of the phase ends of three-phase synchronization winding to be coupled with same-name phase ends of three-phase main generator armature winding through the switching unit and cooperative operation of the voltage synchronizer with the switching unit allows for minimal discrepancy in the frequencies of output voltage of the axial generator and the voltage of external three-phase AC system, which allows meeting one of the necessary requirements to connection of a generator to an external three-phase AC system: synchronization of the output voltage of axial generator with external three-phase AC system.

The minimization of the difference between the frequencies of output voltage of axial generator and voltage of 
external three-phase AC system is provided by the adjustment of the rotor rpm using voltage synchronizer.

The capability of combination of mechanical energy with light energy is achieved through the installation in the SATG case of a PVC, which is coupled with auxiliary single-phase exciter excitation winding enabling the connection to the external PVC, and magnetic gear consisting of the magnetic gear shaft, driver and driven disks and constant magnets mounted on the driver and driven disks with dissimilar poles directed at each other with the driver disk rigidly fixed on the magnetic gear shaft and the driven disk rigidly fixed on the end of the rotor shaft outstanding from the case.

The mechanical rotation energy (torque) is received from external source (e.g., windwheel converting the kinetic energy of wind into mechanical rotation energy) to the magnetic gear shaft and through the magnetic gear transferred to the SATG rotor shaft. During the rotation of SATG rotor, under the torque, the received mechanical energy is converted in the assembly, which includes constant axial multi-polar magnet of subexciter inductor and multi-phase subexciter armature winding, into kinetic energy. In the assembly, which includes main and auxiliary single-phase exciter excitation windings and multi-phase exciter armature winding, the electric energy received from conversion of mechanical energy is combined with electric energy from the PVC converted from light energy.

The synchronized axial two-input generator has extended application sphere due to capability of direct conversion of light energy into DC electric energy and minimal difference between the frequency of SATG output voltage and frequency of external three-phase AC system.

\section{CONCLUSION}

Thus, the solutions suggested by the authors, unlike existing ones, provide the capability of both direct conversion of wind and solar energy into electric energy and connection of the electrotechnical complex built on the basis of developed equipment to the external three-phase $\mathrm{AC}$ system and parallel operation.

The investigation was financially supported by the Russian Foundation for Basic Research and Administration of Krasnodar Krai within scientific project no. 16-48-230500 r_a.

\section{References}

[1] V.A. Butuzov, "Analysis of the world market of helioplants," International scientific journal Alternative energy and ecology, Vol. 2 (142), pp. 95-98, 2014.

[2] A.K. Sokolsky, Wind energy abroad and in Russia - current state and prospects, "Renewable energy sources", Moscow: MSU, 2005.

[3] P.P. Bezrukih, "The state and prospects for the development of renewable energy," Elektrika, Vol. 9, pp. 3-10, 2008.

[4] R. Aubrée, M. Macé, L. Loron, "Design of an efficient small windenergy conversion system with an adaptive sensorless MPPT strategy," Renewable Energy, Vol. 86, pp. 280-291, 2016.

[5] V.S. Krivtsov, A.M. Oleynikov, A.I. Yakovlev, Inexhaustible energy, book 1, Wind power generators, Sevastopol: publ. by Sevastopol National Technical University, 2003.

[6] D.A. Ivashintsev, A.M. Ryzhov, M.V. Kuznetsov, V.N. Kryvoy, N.V. Zuev, Invention Patent of the RF No. 2168062. Wind power generator. 7.12.1999.

[7] S.A. Popov, M.S. Popov, Invention Patent of the RF No. 2643522 Hybrid wind-solar generator, 15.11.2016.

[8] S.A. Popov, Patent of the RF No. 2643522. Wind-solar generator with dual rotor, 30.01.2017.

[9] B.Kh. Gaitov, Ya.M. Kashin, T.B. Gaitova, A.Ya. Kashin, D.V. Paukov, A.V. Goloshchapov, Invention Patent of the RF No. 2450411. Axial two-input contact-less electrical generator machine, 12.01.2011.

[10] B.Kh. Gaitov, T.B. Gaitova, R.Z. Tarshkhoev, Invention Patent of the RF No. 2316877. Method for production of magnetic conductors of axial electrical machines, 10.07.2006.

[11] Ya.M. Kashin, A.Ya. Kashin, L.E. Kopelevich, A.V. Samorodov, M.S. Khristoforov, Invention Patent of the RF No. 2617708. Synchronized axial two-input generator, 17.04.2017. 